\title{
Three-Dimensional CAD-Based Mesh Generator for the Dey-Mittra Conformal FDTD Algorithm
}

\author{
Geoff Waldschmidt and Allen Taflove, Fellow, IEEE
}

\begin{abstract}
It is well-known that the finite-difference time-domain (FDTD) method is subject to significant errors due to the staircasing of surfaces that are not precisely aligned with major grid planes. Dey and Mittra introduced a locally conformal method (D-FDTD) that has shown substantial gains in the accuracy of modeling arbitrary surfaces in the FDTD grid. A mesh generator for this purpose was reported by Yu and Mittra. In this paper, we present the formulation and validation of an alternative CAD-based mesh generator for D-FDTD that has improved capabilities for arbitrary three-dimensional (3-D) perfect electric conductor (PEC) geometries. This mesh generator is capable of importing AutoCad and ProE files of 3-D PEC scatterers and resonators. It can reduce the required FDTD grid resolution by up to 4:1 in each Cartesian direction in 3-D relative to conventional staircased FDTD models when modeling cavity resonances of complex PEC structures such as twisted waveguides.
\end{abstract}

Index Terms-Conformal method, finite-difference time-domain (FDTD), mesh generation.

\section{INTRODUCTION}

$\mathbf{I}$ T IS well documented that the basic finite-difference timedomain (FDTD) method is subject to error due to its simplistic staircasing of geometries [1], [2]. Errors persist even for very small space increments. In the literature, a variety of meshing techniques have been proposed to improve the modeling of arbitrarily shaped geometries in the FDTD grid. Some of these methods include nonorthogonal algorithms and locally conformal algorithms. These methods offer the prospect of modeling arbitrary three-dimensional (3-D) geometries subject to certain limiting considerations.

Unstructured, irregular grids such as those used by finite-element (FE) solvers offer the greatest flexibility for accurate mesh generation. Rather than deforming the geometry to conform to the grid (as in the standard FDTD algorithm), the nonorthogonal mesh conforms to the geometry. A method for implementing nonorthogonal grids for FDTD modeling was reported in [3] and adapted by others [4]-[8]. However, some of these methods suffer from late-time instabilities, regardless of the time step, due to the formulation of the projection vectors [9].

Manuscript received March 17, 2002; revised January 10, 2003. This work was supported by the U.S. Department of Energy, Office of Basic Energy Sciences, under Contract W-31-109-ENG-38.

G. Waldschmidt was with the Electrical and Computer Engineering Department, McCormick School of Engineering, Northwestern University, Evanston, IL 60208 USA. He is now with the Argonne National Laboratory, Argonne, IL 60439 USA (e-mail: waldschm@aps.anl.gov).

A. Taflove is with the Electrical and Computer Engineering Department, McCormick School of Engineering, Northwestern University, Evanston, IL 60208 USA.

Digital Object Identifier 10.1109/TAP.2004.831334
While locally conformal gridding methods do not offer the versatility of nonorthogonal algorithms, they have been rigorously analyzed and have been shown to give superior results to the standard FDTD method [10]. Locally conformal grids are altered only where intersected by the wave-interaction geometry. The grid retains its regular, orthogonal structure with modifications made only locally to specific grid edges and cells. As a result, once the geometry generation is concluded, only slight modifications are needed to existing FDTD codes to implement fully the locally conformal algorithm.

Jurgens et al. introduced the first locally conformal grid for modeling curved perfect electric conductor (PEC) surfaces. This method, referred to as the contour-path FDTD (CP-FDTD) method [11], [12], showed a marked accuracy improvement relative to staircasing methods. Late-time numerical stability problems with CP-FDTD were subsequently overcome by Railton [13], [14]. Recently, Dey and Mittra introduced a new locally conformal method (D-FDTD) [15] that is numerically stable, easier to implement than CP-FDTD, and provides improved accuracy. Yu and Mittra created an automated means of implementing D-FDTD that gives excellent improvement over staircased results [16].

This paper presents the formulation and validation of a CADbased mesh generator that has some improved capabilities relative to that of Yu and Mittra for arbitrary 3-D PEC geometries. The CAD-based mesh generator is capable of importing 3-D geometries from AutoCad and ProE and modeling both scattering and resonating structures using the D-FDTD algorithm. Section II reviews the background of the CP-FDTD and D-FDTD locally conformal algorithms, noting the current merits and limitations of each method. Section III discusses the implementation details of the new CAD-based D-FDTD mesh generator for PEC structures. Finally, Section IV provides and analyzes numerical results.

\section{BACKGROUND OF CP-FDTD AND D-FDTD LOCALLY CONFORMAL ALGORITHMS}

The CP-FDTD and D-FDTD methods [11], [15] are two locally conformal gridding techniques that have been shown to produce substantial improvement in modeling certain types of arbitrary structures. Both methods use the basic orthogonal-grid FDTD algorithm for cells that are not intersected by the waveinteraction geometry, but they distinguish between two classifications of cell/geometry-surface intersections that are unique to each method.

Fig. 1(a) and (b) shows two general classifications of cell intersections for the CP-FDTD method of [11]. The update equa- 


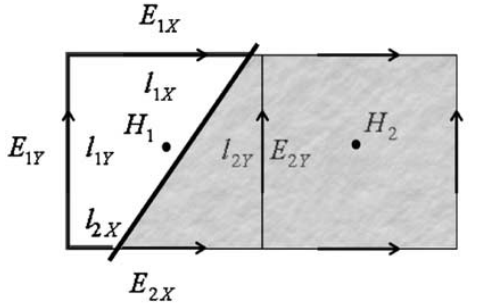

(a)

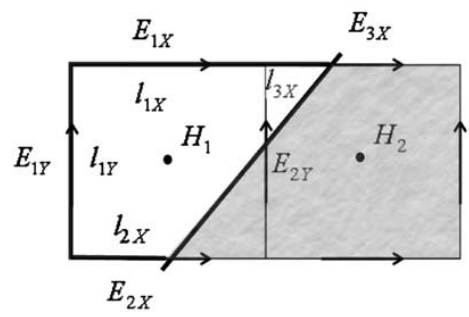

(b)

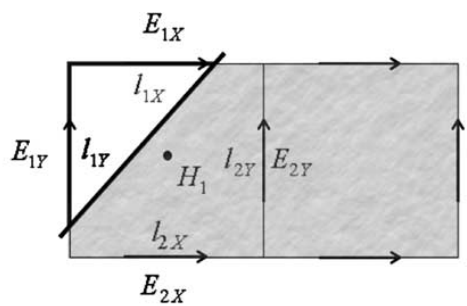

(c)

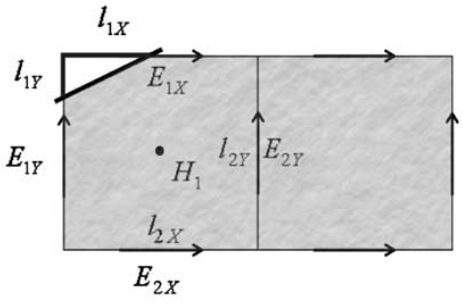

(d)

Fig. 1. General cases for CP-FDTD and D-FDTD locally conformal algorithms. (a), (b) CP-FDTD method where H field is located (a) outside PEC, or (b) inside PEC. (c), (d) D-FDTD method where (c) the stability criteria is met, and (d) the stability criteria is violated.

tion for $H$ depends upon whether the $H$ field is inside or outside the PEC. In Fig. 1(a), $H_{1}$ is outside the PEC so its update equation is the standard circulation of the surrounding $E$ fields. Accounting for the modified edge lengths and areas

$$
\begin{array}{r}
H_{1}^{n+1}=H_{1}^{n}+\frac{\Delta t}{\mu A_{1}}\left(E_{1 Y}^{n+\frac{1}{2}} * l_{1 Y}+E_{1 X}^{n+\frac{1}{2}} * l_{1 X}-E_{2 Y}^{n+\frac{1}{2}}\right. \\
\left.* l_{2 Y}-E_{2 X}^{n+\frac{1}{2}} * l_{2 X}\right) .
\end{array}
$$

In Fig. 1(b), $H_{2}$ is within the PEC. In this case, cell 1 is expanded into cell 2 during the calculation of $H_{1} . H_{2}$ is not updated in cell 2, and the $E$ fields outside the PEC in cell 2 are borrowed from cell 1 . The update equation for this case is as follows:

$$
\begin{aligned}
H_{1}^{n+1}= & H_{1}^{n}+\frac{\Delta t}{\mu\left(A_{1}+A_{2}\right)}\left(E_{1 Y}^{n+\frac{1}{2}} * l_{1 Y}+E_{1 X}^{n+\frac{1}{2}}\right. \\
& \left.*\left(l_{1 X}+l_{3 X}\right)-E_{2 X}^{n+\frac{1}{2}} * l_{2 X}\right) \\
E_{3 X}= & E_{1 X} .
\end{aligned}
$$

Due to field borrowing and cell expansions, this formulation is numerically unstable. An alternate CP-FDTD formulation described in [13], [14] introduces modifications that achieve stability without compromising the accuracy of the solution.

The D-FDTD method is less complex and more stable than the CP-FDTD method since it does not require cell expansion or field borrowing. As shown in Fig. 1(c), (d), D-FDTD imposes stability criteria that require two general classifications of cells depending upon whether the stability criteria are violated.

The D-FDTD stability criteria are based on: 1) the minimum grid-cell face area and 2) the maximum ratio of the grid-cell edge length to the grid-cell face area for a specified cell. If either criterion is not met, the electric fields along the grid-cell face are set to zero. Otherwise the update is treated normally. The actual threshold values for the stability conditions are dependent upon the Courant number. Given a Courant number that is one-half the maximum allowable, D-FDTD requires that the minimum area be $1.5 \%$ of the area in an unmodified grid cell. Second, the ratio of the maximum grid-cell edge length to the area of the

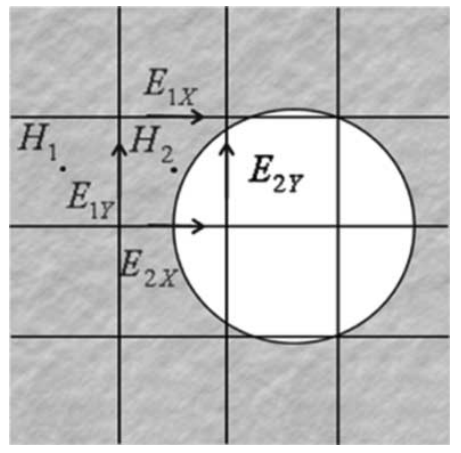

Fig. 2. Field leakage into PEC due to locally conformal algorithm.

grid-cell face must not be more than 15 times greater than that of an unmodified cell.

In Fig. 1(c), all stability considerations have been met for the D-FDTD method. For this case, the $H$ field update equation is identical to the CP-FDTD (1) when the $H$ field is outside the PEC. On the other hand, in Fig. 1(d), the area of interest outside the PEC is below a minimum allowable value, thereby violating one of the stability criterion. The cell is assumed to be entirely filled with a PEC and all cell edge lengths are set equal to zero.

A consequence of the locally conformal methodology is the tendency for numerical fields to propagate into the PEC region. The grid is modified to account for partial edge lengths, but unlike nonorthogonal algorithms, there is not an actual grid entity that lies along the surface of the geometry. Fig. 2, shows the mechanism for field leakage. From the figure, $\mathrm{H}_{2}$ is updated normally according to the D-FDTD algorithm. Assuming the stability requirements are met in that grid face, $\mathrm{H}_{2}$ will be nonzero. The update for $E_{1 Y}$ is as follows:

$$
E_{1 Y}^{n+\frac{1}{2}}=E_{1 Y}^{n-\frac{1}{2}}+\frac{\Delta t}{\varepsilon \Delta x}\left(H_{1}^{n}-H_{2}^{n}\right) .
$$

Since $H_{2}$ is nonzero, $E_{1 Y}$ is nonzero in spite of the fact that it is entirely immersed in a PEC. The numerical energy in the grid is thus able to spread nonphysically outwards into the PEC. 


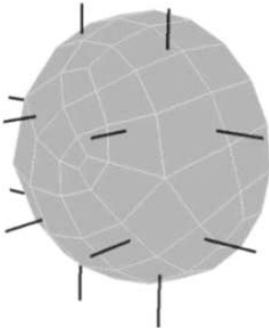

(a)

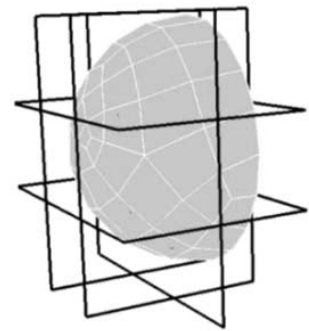

(b)
Fig. 3. FE mesh of a hemisphere. (a) Hemisphere superimposed onto an FDTD grid where the intersected FDTD edges are shown. (b) Electric wall surrounding a hemispherical resonator.

To prevent this leakage from occurring, a PEC shell is wrapped around a resonator as in this case, or a PEC shell is inserted inside a scatterer. The shell is created by simply assigning zero to the electric field edge lengths wherever the shell is needed.

Once the modified areas and edge lengths are determined and the electric wall is created, the D-FDTD algorithm can be implemented easily by existing FDTD solvers. The inclusion of standard FDTD features such as absorbing boundary conditions, total-field/scattered-field surfaces, near-to-far field transformations, and others may be used without alteration. However, the execution time of the D-FDTD method is greater due to the additional multiplications of edge lengths and division by grid-cell areas. For a setup consisting of a six-cell, perfectly matched layer [17] boundary condition, the execution time of the field update equations increased by only $4.3 \%$ as compared with the standard FDTD algorithm.

\section{IMPLEMENTATION DETAILS OF THE CAD-BASED D-FDTD MESH GENERATOR}

A general difficulty in implementing the D-FDTD algorithm in three dimensions is in the systematic ability to create a reliable mesh for an arbitrary geometry. The D-FDTD mesh generator discussed in this paper is capable of robustly importing 3-D PEC geometries from AutoCad and ProE and modeling both scattering and resonating structures. It is built on the FE mesh generator, CUBIT ${ }^{1}$ produced by Sandia National Laboratory [18]. CUBIT produces a conformal nonorthogonal FE mesh consisting of triangular or quadrilateral planar facets. This faceted mesh is imported into the D-FDTD mesh generator where it is superimposed onto a uniform Cartesian FDTD grid. The intersection points of the FE mesh geometry with the FDTD grid are evaluated, and the appropriate FDTD grid-cell edge lengths and areas are modified.

Fig. 3(a) shows a sample FE mesh of a hemispherical scatterer created using CUBIT and superimposed onto an FDTD grid. The FDTD edges that intersect the geometry are shown protruding from the hemisphere. Since this is a scattering geometry, the portions of the edges that are visible in the figure show the modified edge lengths that are calculated by the mesh generator. All other FDTD grid edges are left unchanged. Fig. 3(b)

${ }^{1}$ CUBIT is a registered trademark of Sandia National Laboratory, Albuquerque, NM 87185-0203 USA. illustrates the converse situation where the hemisphere is modeled as a cavity. Here an external electric wall is used to prevent field leakage outside the hemisphere.

Intersections of the faceted FE surface and the local FDTD grid cells must be carefully considered in order to create a general-use mesh generator. To create the D-FDTD mesh, the FE mesh must first be located in the standard FDTD grid away from any total field/scattered field boundaries and absorbing boundaries. Each facet of the FE mesh is evaluated separately, and the edges of the FDTD grid are surveyed in order to determine if an intersection exists. The listing of the coordinates of each facet produced by the FE mesh generator create either a clockwise or counterclockwise rotation. This orientation is used to determine the interior and exterior regions of the wave interaction region.

The procedure for evaluating an intersection of a facet with the FDTD grid begins by locating the FDTD grid edges near the FE facet. In the case of an $x$-directed FDTD edge (a similar methodology may be applied to $y$ - and $z$-directed edges), it is determined whether its $(y, z)$ coordinates are positioned internally to the facet. Each edge of the facet is used to separately test for inclusion of the $(y, z)$ coordinates of the FDTD grid edge in its interior. The cross-products of the vector representation of the edges of the facet are taken with the normal vector of the facet such that the resultant inclusion vector is directed inside the facet. The edge vectors are then extended out in both directions and formed into a plane by extruding the line into the $\pm x$ directions. The $(y, z)$ coordinate of the $x$-directed FDTD grid edge is evaluated to determine if it lies in the direction of the inclusion vectors relative to the half planes. If it does for all edges of the facet, the FDTD $x$-directed grid edge is determined to be located within the interior of the facet extruded in the $\pm x$ directions; otherwise, it is not.

It has not yet been determined whether an actual intersection has occurred. The plane equation for the FE facet is used to evaluate the $x$ value of the facet at the coordinate $(y, z)$. An intersection of the $x$-directed FDTD edge with the facet will occur if the $x$-value of the facet is determined to be within the span of the FDTD edge. The interior of the wave-interaction region is determined from the direction of the circulation of the coordinates of the facet produced by CUBIT. The edge length can be determined using the portion of the FDTD edge inside this region, and the electric wall can be built exterior to this region.

Fig. 4 shows problematic cases that can often occur for arbitrary geometries. Fig. 4(a) shows a special case where a facet of the FE mesh intersects an FDTD edge at a vertex. The division of the grid edges inside and outside the wave-interaction region can be determined normally once the edges contacting the intersected vertex are evaluated. The top leftmost grid edge is completely outside the wave-interaction region while the central vertical grid edge is completely within, and the top rightmost edge is considered to be doubly intersected. Once this evaluation is made, the edge length and area calculation of the FDTD grid cells proceeds normally.

These intersections are all termed point-wise since the FDTD edge intersects the FE mesh at a single location. On the other hand, planar intersections occur when an FDTD edge lies directly along the surface of the FE mesh as in Fig. 4(b). Planar intersections do not divide an FDTD grid edge at a single point 


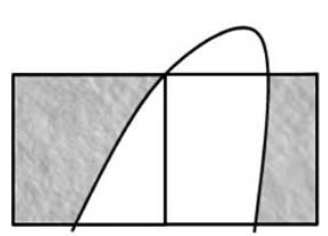

(a)

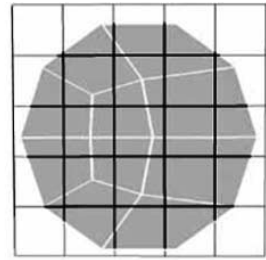

(b)
Fig. 4. Special cases concerning the intersection of the FE mesh with FDTD grid including (a) intersection of FE mesh at a vertex of the FDTD grid; (b) planar intersections of the FE mesh with the FDTD grid. In (b), the facets of the FE mesh are outlined in white and the intersected FDTD grid edges internal to the geometry are emphasized with black.

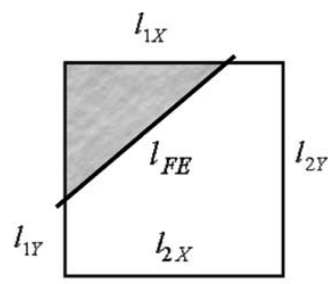

(a)

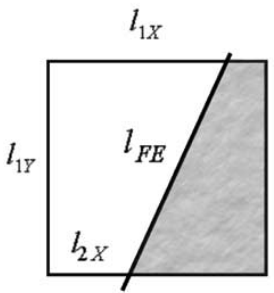

(b)

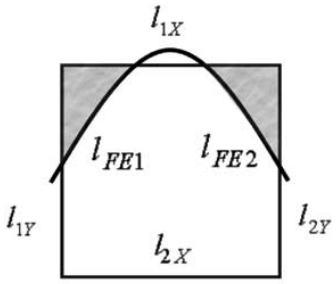

(c)

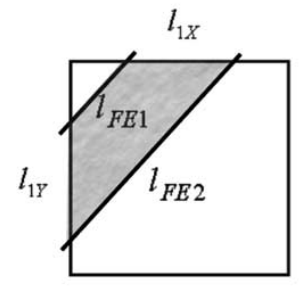

(d)
Fig. 5. Possible intersections of the FE mesh with the FDTD grid. Grid-cell areas are calculated based on the resultant edge lengths.

into regions inside and outside the PEC as for point-wise intersections. Instead, the length of the FDTD edge in the wave-interaction region must be evaluated after determining all the intersections of the FE facets with the FDTD edge since a single FDTD edge may be intersected multiple times. After compilation, the portion of the FDTD edges outside the facets are considered to be in the wave-interaction region since the facets represent the PEC boundary of the geometry.

The planar intersection points are not found by representing the facet by a planar equation as for point-wise intersections. Rather the equation of a line passing through each edge of the FE facet is used. Assuming an $x$-directed FDTD edge at $\left(y_{0}, z_{0}\right)$ in the $x y$ plane, the $x$ value of each of the edges of the FE facet at $y=y_{0}$ are calculated by representing the edges as infinitely extending lines. If the $x$ value lies on the FDTD edge, then an intersection point is found. Since multiple facets may intersect the same edge, the intersections must be grouped to determine FDTD edge lengths once all intersections of FE facets with FDTD edges have been determined.

Once all the point-wise and planar mesh geometry is compiled, special consideration must be given to those edges that contain both classifications of intersections. During the final analysis of these types of edges, the interior and exterior regions of the PEC must be compared and a final evaluation made based on the geometry of the hybrid intersection point.

Fig. 5 provides a sampling of typical point-wise intersections that can occur and are accounted for in this mesh generator. This figure shows examples of intersections of the faceted FE mesh with Cartesian FDTD grid-cell faces that demonstrate singly intersected edges, doubly intersected edges, and multiple doubly intersected edges. If an edge is singly intersected, as in Fig. 5(a) and (b), the calculation of the edge length is found by simply determining the edge length outside the PEC. For multiply intersected edges as shown in Fig. 5(c) and (d), it is determined whether the region between the intersection points or away from them is outside the PEC. In Fig. 5(c), the PEC is external to the intersection points, and the grid-cell edge length becomes the interior segment length between the two points. In Fig. 5(d), the PEC is between the intersection points and the grid-cell edge length becomes the addition of the segment lengths to either side of the intersecting curve.

Once the edge lengths of the FDTD grid are known, the area can be calculated. Fig. 5(a) shows a simple case where two adjacent edges of an FDTD cell are intersected. The area of this cell is simply the area of the triangular PEC wedge subtracted from the full unmodified cell area. Similarly, Fig. 5(b) consists of two singly intersected edges, but these edges are not adjacent. The area is then the sum of two cross products as follows:

$$
A=\frac{l_{1 X} \times l_{1 Y}+l_{2 X} \times l_{\mathrm{FE}}}{2} .
$$

Fig. 5(c), shows the case where an edge is doubly intersected and two other edges are singly intersected. Each pair of intersection points must be matched appropriately. To avoid overlap and unnecessary complexity, it is useful to calculate the area inside the PEC in this case so that the cell may be effectively divided into two regions independent of each other. The area of the two portions of the PEC may then be subtracted from the total area of an unmodified cell to determine the total area in the cell. In Fig. 5(d), the sum of the cross products of the vector edge lengths spanning the interior of the doubly intersected edges must be determined in order to find the area of the cell. Since the PEC is inside this region, the final area is found by subtracting this from the area of an unmodified cell.

\section{NUMERICAl ReSUlTS AND APPLICATIONS}

The accuracy of the CAD-based D-FDTD mesh generator has been determined via comparison with published results in addition to comparison with Ansoft High Frequency Structure Simulator (HFSS)2 [19], a commercial FE method solver. The first validation sequence compared results published by Railton and Schneider for the resonant frequencies of cylindrical and spherical cavities [10]. Fig. 6 shows a cut-away of the FE mesh of a sample cylindrical resonator used in this study. FDTD edges that are intersected by the FE mesh are drawn showing their partial edge lengths and subsequently modified grid-cell areas. Fig. 7(a), taken from Railton and Schneider [10], shows the error of the standard staircasing FDTD algorithm compared with the stabilized CP-FDTD and D-FDTD methods at a resolution of $\lambda / 20$ for cylindrical cavities oriented at various angles. Fig. 7(b) shows the error of Railton and Schneider's D-FDTD results compared with the error using the new D-FDTD CAD-based mesh generator. The results for the new D-FDTD mesh generator are within the error limits reported in [10]. Differences in

\footnotetext{
USA.

${ }^{2}$ HFSS is a registered trademark of Ansoft Corporation, Pittsburgh, PA 15090
} 


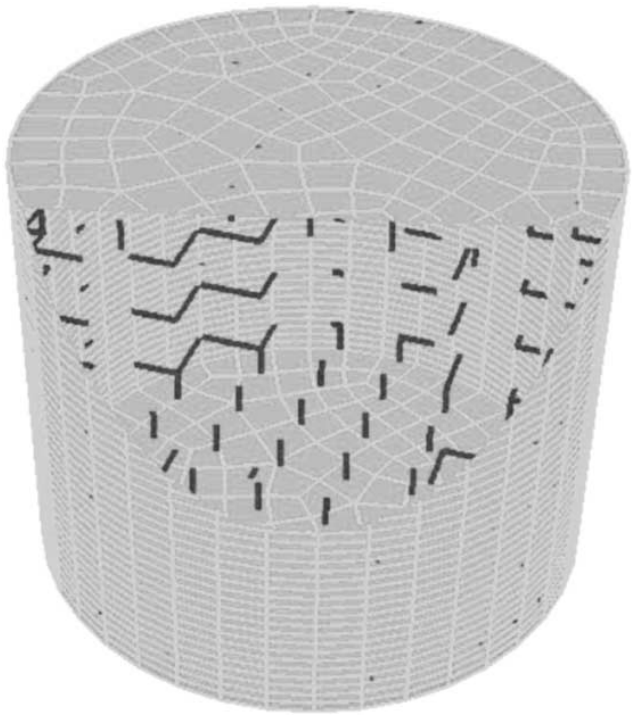

Fig. 6. FE mesh of the cylindrical cavity resonator in the FDTD grid.

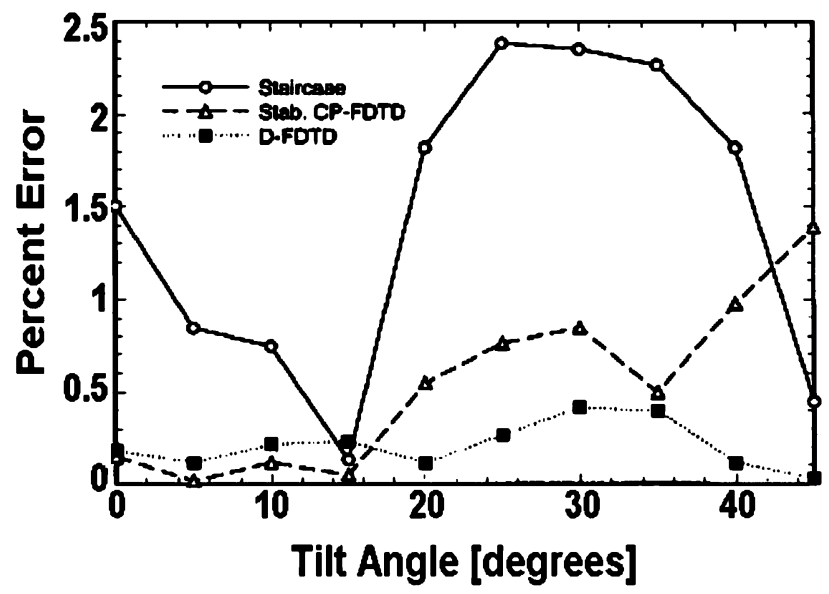

(a)

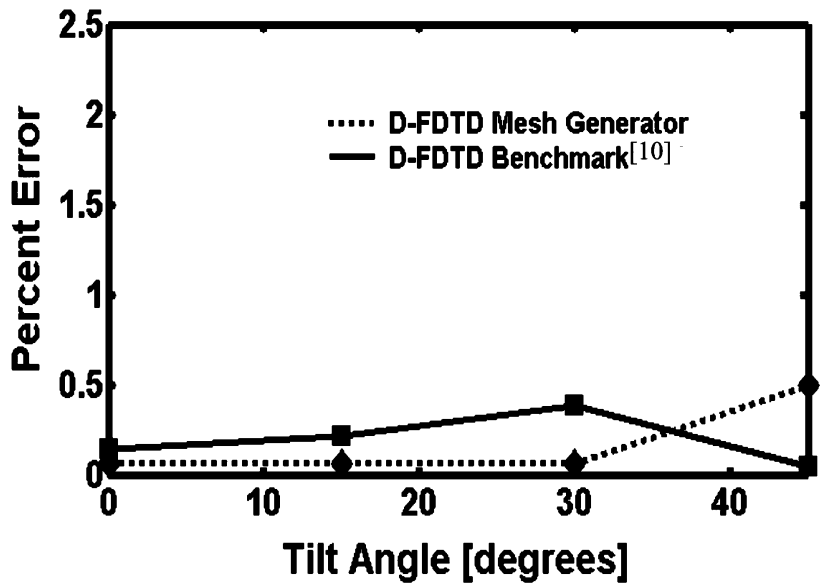

(b)

Fig. 7. Comparison of error in the resonant frequency versus tilt angle of the cylindrical cavity of Fig. 6 for (a) staircase FDTD, stabilized CP-FDTD, and D-FDTD methods, as reported in [10] and (b) D-FDTD method with the new CAD-based mesh generator.

the exact values are likely a consequence of the random placement of the geometry in the grid.

Fig. 8, shows a cut-away of the FE mesh of a sample spherical resonator as represented in its entirety in the D-FDTD grid. The

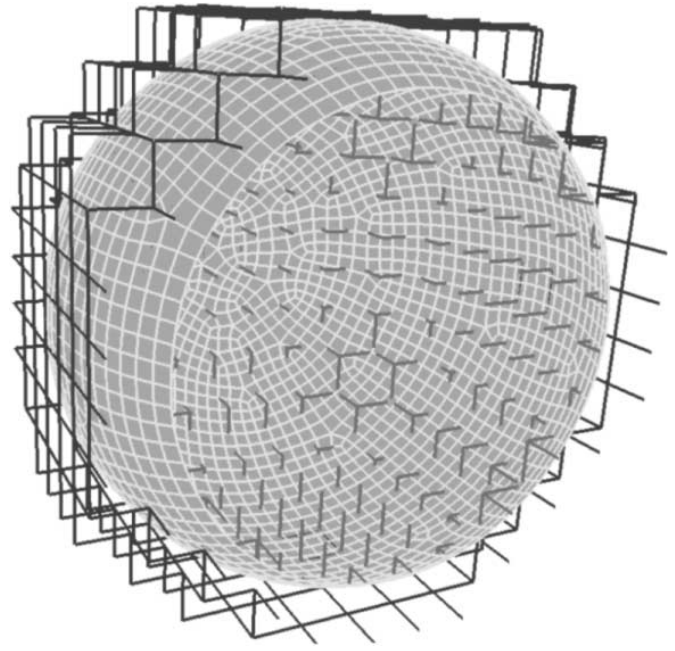

Fig. 8. FE mesh of the spherical cavity resonator in the FDTD grid.

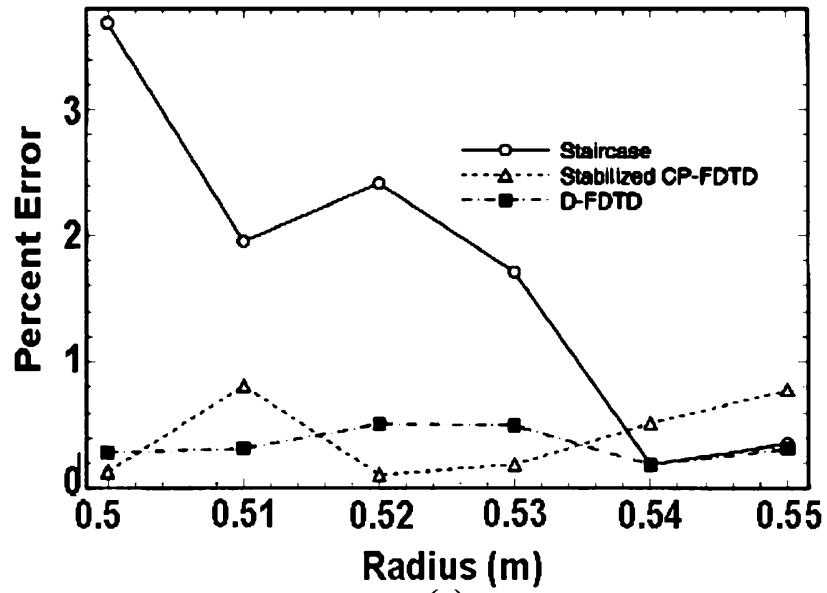

(a)

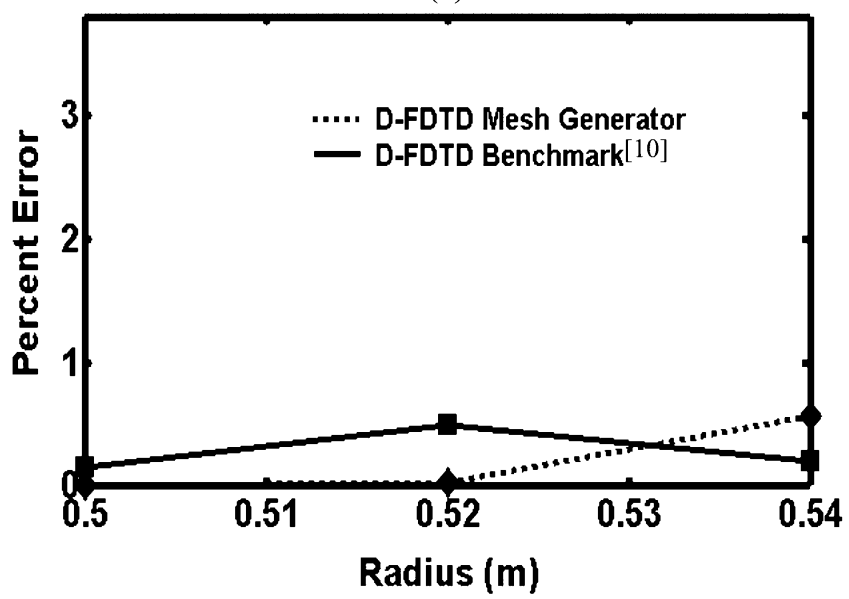

(b)

Fig. 9. Comparison of error in the resonant frequency versus radius of the spherical cavity of Fig. 8 for (a) staircase FDTD, stabilized CP-FDTD, and D-FDTD methods, as reported in [10] and (b) D-FDTD method with the new CAD-based mesh generator.

shell surrounding the sphere is explicitly shown in Fig. 8 in addition to the partial edge lengths of the intersected FDTD grid edges interior to the sphere. Fig. 9(a), taken from Railton and Schneider [10], again shows the error of the staircasing FDTD 


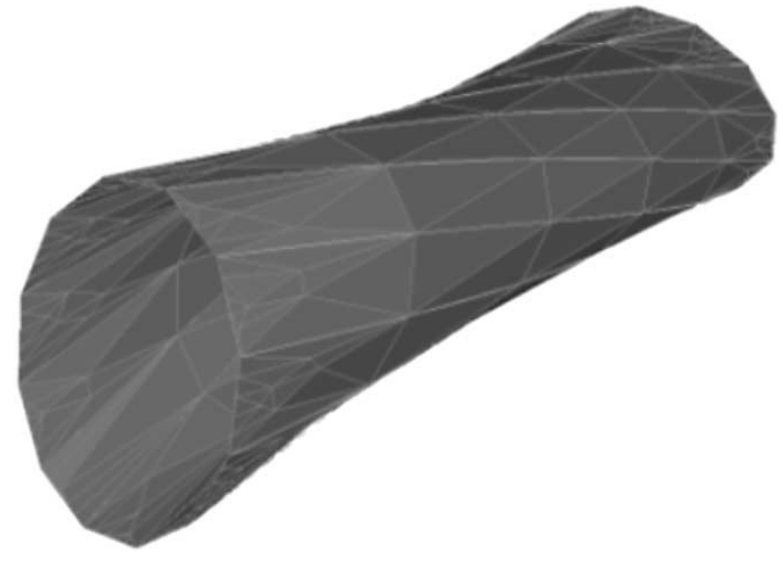

Fig. 10. FE mesh of a resonant cavity formed by a twisted waveguide of elliptical cross section.

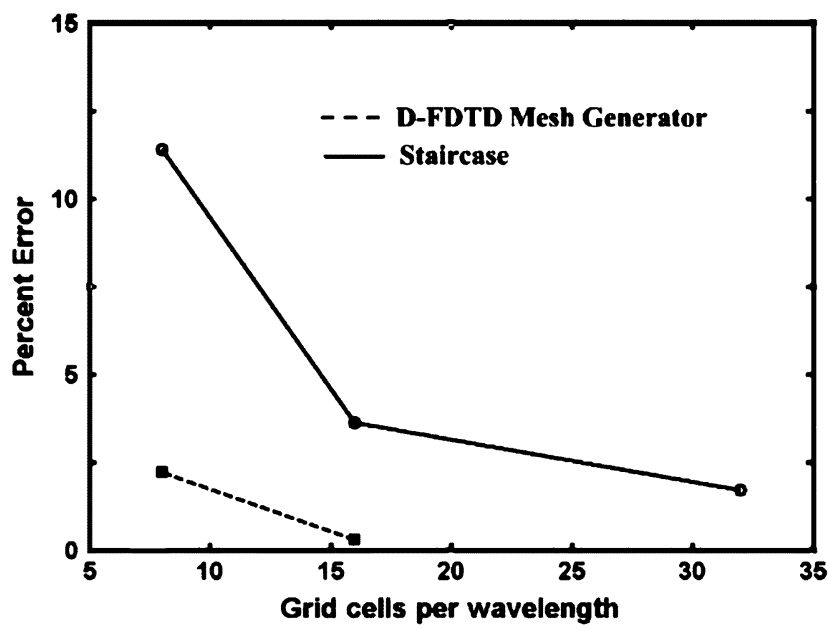

Fig. 11. Comparison of the error in the resonant frequency of the fundamental mode of the twisted elliptical waveguide cavity of Fig. 10 obtained using the staircased FDTD algorithm and the new CAD-based D-FDTD algorithm.

algorithm compared with the stabilized CP-FDTD and D-FDTD methods, this time at a resolution of $\lambda / 10$. Fig. 9(b), shows the error of Railton and Schneider's D-FDTD results compared with the error using the new D-FDTD mesh generator. Once again the results of the new D-FDTD mesh generator are within the error limits reported in [10].

Additionally, the D-FDTD mesh generator was used to analyze the resonant frequency of a cavity comprised of a twisted waveguide of elliptical cross section. Here, the benchmark solution is taken to be a highly resolved FE method calculation using Ansoft HFSS. Figs. 10 and 11, show the geometry of this resonator and a comparison of the error in the calculated resonant frequency of the D-FDTD model versus the staircased FDTD model. We see that the new mesh generator for D-FDTD yields an error at eight grid cells per wavelength that is comparable to that obtained using the conventional staircased algorithm at 32-grid cells per wavelength.

\section{SUMmARY AND CONCLUSION}

This paper has introduced a new CAD-based mesh generator for the Dey-Mittra conformal FDTD algorithm (D-FDTD) that is capable of robustly and automatically importing AutoCad and ProE files for FE models of PEC scatterers and resonators. The new mesh generator produces results comparable to previously published solutions using the D-FDTD methodology and to benchmark FE frequency-domain software such as Ansoft HFSS. The new mesh generator for D-FDTD can reduce the required FDTD grid resolution by up to $4: 1$ in each Cartesian direction in 3-D relative to conventional staircased FDTD models when modeling cavity resonances of complex PEC structures such as twisted waveguides.

\section{REFERENCES}

[1] A. C. Cangellaris and D. B. Wright, "Analysis of the numerical error caused by the stair-stepped approximation of a conducting boundary in FDTD simulations of electromagnetic phenomena," IEEE Trans. Antennas Propagat., vol. 39, pp. 1518-1525, Oct. 1991.

[2] R. Holland, "Pitfalls of staircase meshing," IEEE Trans. Electromagn. Compat., vol. 35, pp. 434-439, Nov. 1993.

[3] — "Finite difference solutions of Maxwell's equations in generalized nonorthogonal coordinates," IEEE Trans. Nucl. Sci., vol. NS-30, pp. 4589-4591, 1983.

[4] N. Madsen, "Divergence preserving discrete surface integral methods for Maxwell's equations using nonorthogonal unstructured grids," $J$. Comput. Phys., vol. 119, pp. 34-45, 1995.

[5] M. Fusco, "FDTD algorithm in curvilinear coordinates," IEEE Trans. Antennas Propagat., vol. 38, pp. 76-89, Jan. 1990.

[6] J. F. Lee, R. Palandech, and R. Mittra, "Modeling three-dimensional discontinuities in waveguides using nonorthogonal FDTD algorithm," IEEE Trans. Microwave Theory Tech., vol. 40, pp. 346-352, Feb. 1992.

[7] Y. Hao and C. J. Railton, "Analyzing electromagnetic structures with curved boundaries on Cartesian FDTD meshes," IEEE Trans. Microwave Theory Tech., vol. 46, pp. 82-88, Jan. 1998.

[8] S. D. Gedney, F. Lansing, and D. Rascoe, "A full-wave analysis of passive monolithic integrated circuit devices using a generalized Yee-algorithm," IEEE Trans. Microwave Theory Tech., vol. 44, pp. 1393-1400, Aug. 1996.

[9] S. D. Gedney and J. A. Roden, "Numerical stability of nonorthogonal FDTD methods," IEEE Trans. Antennas Propagat., vol. 48, pp. 231-239, Feb. 2000.

[10] C. J. Railton and J. B. Schneider, "An analytical and numerical analysis of several locally conformal FDTD schemes," IEEE Trans. Microwave Theory Tech., vol. 47, pp. 56-66, Jan. 1999.

[11] T. G. Jurgens, A. Taflove, K. Umashankar, and T. G. Moore, "Finite-difference time-domain modeling of curved surfaces," IEEE Trans. Antennas Propat., vol. 41, pp. 1703-1708, Dec. 1993.

[12] T. G. Jurgens and A. Taflove, "Three-dimensional contour FDTD modeling of scattering from single and multiple bodies," IEEE Trans. Antennas Propagat., vol. 41, pp. 1703-1708, Dec. 1993.

[13] C. J. Railton, I. J. Craddock, and J. B. Schneider, "Improved locally distorted CPFDTD algorithm with provable stability," Electron. Lett., vol. 31, no. 18, Aug. 1995.

[14] C. J. Railton and I. J. Craddock, "A modified CPFDTD algorithm for the analysis of arbitrary 3D PEC structures," in Proc. Inst. Elect. Eng., vol. 143, Oct. 1996, pp. 367-372.

[15] S. Dey and R. Mittra, "A locally conformal finite-difference time-domain (FDTD) algorithm for modeling three-dimensional perfectly conducting objects," IEEE Microwave Guided Wave Lett., vol. 7, pp. 273-275, Sept. 1997.

[16] W. Yu and R. Mittra, "A conformal FDTD software package modeling antennas and microstrip circuit components," IEEE Trans. Antennas Propagat., vol. 42, pp. 28-39, Oct. 2000.

[17] J. P. Berenger, "A perfectly matched layer for the absorption of electromagnetic waves," J. Comput. Phys., vol. 114, pp. 185-200, 1994.

[18] R. Leland, CUBIT Development Team, Sandia National Lab., Albuquerque, NM, "Cubit mesh generation environment," User's Manual, Version 6.0, vol. 1, 2001.

[19] Ansoft HFSS, 2001. Version 8.0. 


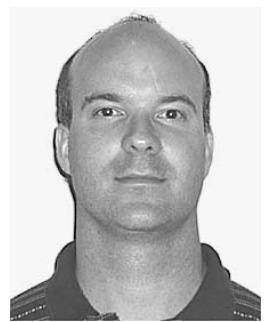

Geoff Waldschmidt was born in Waukegan, IL, on January 15, 1969. He received the B.S. degree in computer science from Lake Forest College, Lake Forest, IL, in 1991 and the M.S. and Ph.D. degrees in electrical engineering from Northwestern University, Evanston, IL, in 2000 and 2002, respectively.

From 1996 to 2002, he was a laboratory Graduate Student at Argonne National Laboratory, Evanston, IL, where he engaged in research and diagnostic work for the Radio Frequency Group at the Advanced Photon Source, a commercially funded accelerator facility used by industry to perform high flux, high resolution X-ray experiments. He also worked on the design of a novel, cost effective linear accelerator and next generation mm-wave accelerators. Since 2002, he has been an RF Engineer at Argonne where he is involved in the design and development of RF systems in the storage ring and linear accelerator.

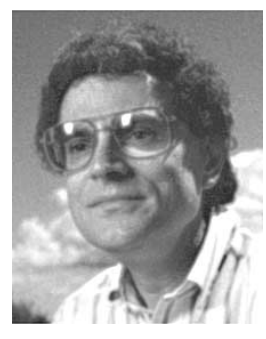

Allen Taflove (F'90) was born in Chicago, IL, on June 14, 1949. He received the B.S., M.S., and Ph.D. degrees in electrical engineering from Northwestern University, Evanston, IL, in 1971, 1972, and 1975, respectively.

After nine years as a Research Engineer at the IIT Research Institute, Chicago, IL, he returned to Northwestern in 1984. Since 1988, he has been a Professor in the Department of Electrical and Computer Engineering, McCormick School of Engineering, Northwestern. Since 1972, he has pioneered basic theoretical approaches and engineering applications of finite-difference time-domain (FDTD) computational electrodynamics. He coined the FDTD acronym in a 1980 IEEE paper, and in 1990 was the first person to be named a Fellow of IEEE in the FDTD area. Currently, FDTD is one of the most powerful and widely used methods for solving Maxwell's equations to model linear and nonlinear electromagnetic wave interactions with electrically large and complex structures. In 1995, he authored a popular textbook on this subject Computational Electrodynamics: The Finite-Difference Time-Domain Method (Boston, MA: Artech House, 1995), which is now in its second edition (2000), with a third edition is planned for 2005. In total, he has authored or co-authored four books, 14 invited papers or chapters in books, 79 refereed journal papers, approximately 200 conference papers and abstracts, and 14 U.S. patents. These publications resulted in his being named to the Highly Cited Researchers list of the Institute for Scientific Information. He has been the thesis adviser of $16 \mathrm{Ph} . \mathrm{D}$. recipients who hold professorial or technical staff positions at major institutions including research universities and national labs. Currently, he serves as the faculty Master of Northwestern's 140-student Slivka Residential College of Science and Engineering. He also serves as the Faculty Advisor to Northwestern's Undergraduate Design Competition, the student chapters of the Eta Kappa Nu and Tau Beta Pi engineering honor societies, and McCormick's Honors Program in Undergraduate Research. His efforts on behalf of students at all levels were recognized by Northwestern when he was named a C. D. McCormick Professor of Teaching Excellence in 2000. His research interests span much of the electromagnetic spectrum. He and his students are currently modeling electrodynamic phenomena ranging from geophysically induced extremely low frequency wave propagation about the entire Earth to the lasing behavior of aggregates of micron-scale zinc-oxide particles exhibiting four-level quantum-system characteristics. The principle that "Maxwell's equations work from de to light" is vividly demonstrated in his laboratory every day. 